\title{
Mantle Convection Modeling with Viscoelastic/Brittle Lithosphere: Numerical and Computational Methodology
}

\author{
Louis Moresi ${ }^{1}$, David May ${ }^{2}$, Justin Freeman $^{1}$, and Bill Appelbe ${ }^{2}$ \\ 1 Monash University, Dept. of Mathematical Science \\ Clayton, Victoria 3186, Australia \\ \{Louis.Moresi, Justin.Freeman\}@sci.monash. edu.au \\ http://wasabi.maths.monash.edu/MonashClusterComputing \\ ${ }^{2}$ Victorian Partnership for Advanced Computing \\ Carlton South, Victoria 3053, Australia \\ \{davidm, bill\}@vpac.org \\ http://www.vpac.org/snark
}

\begin{abstract}
The Earth's tectonic plates are strong, viscoelastic shells which make up the outermost part of a thermally convecting, predominantly viscous layer; at the boundaries between plates the rheology is thought to be dominated by brittle processes. Brittle failure of the lithosphere occurs when stresses are high. In order to build a realistic simulation of the planet's evolution, the complete viscoelastic / brittle convection system needs to be considered. A Lagrangian Integration point finite element method is discussed which can simulate very large deformation viscoelasticity with a strain-dependent yield stress. We also describe the general, parallel implementation of this method (SNARK) and compare the performance to a highly optimized, serial prototype code (ELLIPSIS). The specialized code shows better scaling for a single processor. The parallel scaling of the general code is very flat for "realistic" problem sizes indicating efficient use of multiple processors.
\end{abstract}

\section{Introduction}

Solid state convection in the Earth's mantle drives the surface motion of a cool thermal boundary layer comprising a number of distinct lithospheric plates. Motions in the mantle are described by the equations of fluid dynamics. The rheology needed to describe deformation in the lithosphere is highly non-linear, and, in addition, near the surface where temperatures are less than $\approx 600^{\circ} \mathrm{C}$ it also becomes necessary to consider the role of elasticity (Watts et al, 1980). The strong correlation between seismicity and plate boundaries (e.g. Barazangi \& Dorman, 1969) makes it seem likely that plate motions are associated with localization of deformation occuring when stresses reach the yield strength of the lithosphere. This suggests that the fundamental process is thermal convection; plate tectonics is the manner in which the system organizes. Having identified the need for efficient, large-scale convection simulations with elastic effects in an evolving cool lithosphere, we present a method for simulating viscoelastic/brittle materials during very large deformation, and describe the practical implementation of this method into software for parallel cluster architectures. 


\section{Mathematical Model}

We begin with the classical momentum conservation equation:

$$
\nabla \cdot \sigma=f
$$

where $\boldsymbol{\sigma}$ is the stress tensor and $\boldsymbol{f}$ a force term. As we are interested only in very slow deformations of highly viscous materials, (infinite Prandlt number) we have neglected all inertial terms in (1), and assume incompressible flow.

We employ a Maxwell viscoelastic model which assumes that the strain rate tensor, $D$, defined as:

$$
D_{i j}=\frac{1}{2}\left(\frac{\partial V_{i}}{\partial x_{j}}+\frac{\partial V_{j}}{\partial x_{i}}\right)
$$

is the sum of an elastic strain rate tensor $\boldsymbol{D}_{e}$ and a viscous strain rate tensor $\boldsymbol{D}_{v}$. The velocity vector, $\boldsymbol{V}$, is the fundamental unknown of our problem and all these entities are expressed in the fixed reference frame $x_{i}$.

To account for the low-temperature lithospheric rheology, brittle behaviour has been parameterized using a non-linear effective viscosity. To determine the effective viscosity we introduce a Prandtl-Reuss flow rule for the plastic part of the stretching, and introducing another additive term in the stretching $D_{p}$ :

$$
\frac{\nabla}{2 \mu}+\frac{\boldsymbol{\tau}}{2 \eta}+\lambda \frac{\boldsymbol{\tau}}{2|\boldsymbol{\tau}|}=\boldsymbol{D}_{e}+\boldsymbol{D}_{v}+\boldsymbol{D}_{p}
$$

where $\lambda$ is a parameter to be determined such that the stress remains on the yield surface, and $|\boldsymbol{\tau}| \equiv\left(\tau_{i j} \tau_{i j} / 2\right)^{(1 / 2)}$. The plastic flow rule introduces a non-linearity into the constitutive law which, in general, requires iteration to determine the equilibrium state.

$\stackrel{\nabla}{\tau}$ is the Jaumann corotatonal stress rate for an element of the continuum, $\mu$ is the shear modulus and $\eta$ is shear viscosity. The Jaumann stress rate is the objective (frame invariant) quantity given by

$$
\stackrel{\nabla}{\tau}=\frac{D \boldsymbol{\tau}}{D t}+\boldsymbol{\tau} \mathbf{W}-\mathbf{W} \boldsymbol{\tau}
$$

where $\mathbf{W}$ is the material spin tensor.

In the mantle, the force term from equation (1) is a gravitational body force due to density changes. We assume that these arise, for any given material, through temperature effects:

$$
\nabla \cdot \boldsymbol{\tau}-\nabla p=g \rho_{0}\left(1-\alpha_{T} T\right) \hat{\mathbf{z}}
$$

where $g$ is the acceleration due to gravity, $\rho_{0}$ is material density at a reference temperature, $\alpha_{T}$ is the coefficient of thermal expansivity, and $T$ is temperature. $\hat{\mathbf{z}}$ is a unit vector in the vertical direction. We have also assumed that the variation in density only needs to be considered in the driving term (the Boussinesq approximation). 
The energy equation governs the evolution of the temperature in response to advection and diffusion of heat through the fluid:

$$
\frac{D T}{D t}=-\kappa \nabla^{2} T
$$

where $\kappa$ is the thermal diffusivity of the material.

The viscosity of the mantle at long timescale is known to be a complicated function of temperature, pressure, stress, grain-size, composition (particularly water content) etc (Karato \& Wu, 1993). Despite this complexity, the dominant effect on the viscosity from the point of view of the large-scale dynamics of the system is the effect of temperature (e.g. Solomatov, 1995).

\subsection{Numerical Implementation}

As we are interested in solutions where very large deformations may occur - including thermally driven fluid convection, we would like to work with a fluid-like system of equations. Hence we obtain a stress / strain-rate relation by expressing the Jaumann stress-rate in a difference form:

$$
\stackrel{\nabla}{\boldsymbol{\tau}} \approx \frac{\boldsymbol{\tau}^{t+\Delta t^{e}}-\boldsymbol{\tau}^{t}}{\Delta t^{e}}-\mathbf{W}^{t} \boldsymbol{\tau}^{t}+\boldsymbol{\tau}^{t} \mathbf{W}^{t}
$$

where the superscripts $t, t+\Delta t^{e}$ indicate values at the current and future timestep respectively.

The equation of motion is then

$$
\nabla\left(\eta_{\mathrm{eff}} \mathbf{D}^{t+\Delta t^{e}}\right)-\nabla p=g \rho_{0}\left(1-\alpha_{T} T\right) \hat{\mathbf{z}}-\nabla\left(\eta_{\mathrm{eff}}\left[\frac{\boldsymbol{\tau}^{t}}{\mu \Delta t^{e}}+\frac{\mathbf{W}^{t} \boldsymbol{\tau}^{t}}{\mu}-\frac{\boldsymbol{\tau}^{t} \mathbf{W}^{t}}{\mu}\right]\right)
$$

The velocity field $\mathbf{u}$ and pressure at $t+\Delta t^{e}$ can be solved for a given temperature distribution and the stress history from the previous step. Our system of equations is thus composed of a quasi-viscous part with modified material parameters and a right-handside term depending on values from the previous timestep. This approach minimizes the modification to the viscous flow code. Instead of using physical values for viscosity we use effective material properties (8) to take into account elasticity through a softening of the viscous term.

\section{Computational Method}

Having devised a suitable mathematical representation of the class of problems we wish to model, we need to choose a numerical algorithm which can obtain an accurate solution for a wide range of conditions. Our method is based closely on the standard finite element method, and is a direct development of the Material Point Method of Sulsky et al. (1995). Our particular formulation could best be described as a finite element representation of the equations of fluid dynamics with moving integration points. 
A mesh is used to discretize the domain into elements, and the shape functions interpolate node points in the mesh in the usual fashion. Material points embedded in the fluid are advected using the nodal point velocity field interpolated by the shape functions.

The problem is formulated in a weak form to give an integral equation, and the shape function expansion produces a discrete (matrix) equation. Equation (1) in weak form becomes

$$
\int_{\Omega} N_{(i, j)} \tau_{i j} d \Omega-\int_{\Omega} N_{, i} p d \Omega=\int_{\Omega} N_{i} f_{i} d \Omega
$$

where $\Omega$ is the problem domain, and the trial functions, $N$, are the shape functions defined by the mesh; we have assumed no non-zero traction boundary conditions are present. For the discretized problem, these integrals occur over subdomains (elements) and are calculated by summation over a finite number of sample points within each element. For example, in order to integrate a quantity, $\phi$ over the element domain $\Omega^{e}$ we replace the continuous integral by a summation

$$
\int_{\Omega^{e}} \phi d \Omega \leftarrow \sum_{p} w_{p} \phi\left(\mathbf{x}_{p}\right)
$$

In standard finite elements, the positions of the sample points, $\mathbf{x}_{p}$, and the weighting, $w_{p}$ are optimized in advance. In our scheme, the $\mathbf{x}_{p}$ 's correspond precisely to the Lagrangian points embedded in the fluid, and $w_{p}$ must be recalculated at the end of a timestep for the new configuration of particles.

\section{Efficient Parallel Implementation}

One advantage of the Lagrangian integration point FEM is that it permits the use of a grid which is significantly more regular than the geometrical complexity developed by the model. In a parallel code this has the advantage that the mesh connectivity and domain decomposition is either static or evolves much more slowly than the Lagrangian particle distribution. This fact leads to considerable efficiencies in the allocation and access of distributed global matrices.

In the original, serial implementation of the method (ELLIPSIS, as described in Moresi et al, 2003) a specialized multigrid velocity solver specifically optimized for geoscience problems (Moresi and Solomatov, 1995) was used. This multigrid solver was coupled to a preconditioned Uzawa iteration for pressure (Moresi and Solomatov, 1995) which was found to be robust for strong variations in material properties.

In the parallel code (SNARK), we sought a more general implementation which would be suitable for a broader range of problems, with a more flexible code design (See the paper by Appelbe et al in this volume). The PETSc framework was chosen as the platform since it provides both the interface for domain-decomposition, parallel vector / matrix storage, and a wide range of solution methods. PETSc makes it possible to switch between the many different included solvers very easily. For the velocity solver we found the included GMRES solver to be a reliable starting point for most problems, coupled to the same preconditioned Uzawa scheme developed for the specialized problem. 
In this section we demonstrate the impact on solver efficiency of choosing a general approach over a highly specialized one.

\subsection{Typical Problem}
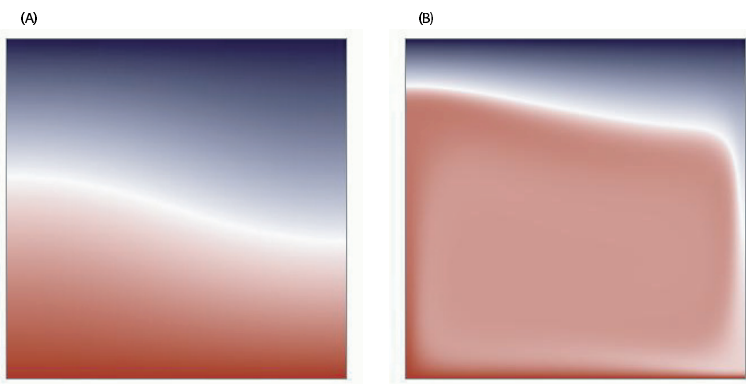

Fig. 1. Convection problem used for timings. The initial condition is on the left (A), and the final, steady-state temperature solution is on the right (B). Blue (dark) is cool, Red (mid-gray) is warm

We benchmark the ELLIPSIS and SNARK codes using a two dimensional convection problem in a $1 \times 1$ box of $64 \times 64$ elements, temperature fixed top $(T=0)$ and bottom $(T=1)$, free slip boundaries, Rayleigh number $10^{6}$, and initial condition

$$
T(x, y)=(1-y)+(\cos \pi x \cdot \sin \pi y) / 100
$$

and using a viscosity dependent on temperature:

$$
\eta=\eta_{0} \exp \left(\frac{E}{T+T_{0}}\right)
$$

In which $T_{0}$ was chosen to be 1 , and $E, \eta_{0}$ were chosen to give a basal viscosity of 1 , and a viscosity contrast of $10^{9}\left(E=41.44, \eta_{0}=10^{-9}\right)$

A thermal convection cell develops as shown in figure 1. The temperature dependence of viscosity leads to a thick, cool upper thermal boundary layer, with a vigorously convecting layer beneath it. Strong viscosity gradients develop in the thermal boundary layers which may influence the convergence rate of the solver. (See Moresi et al, 1996) In the case of the ELLIPSIS multigrid and the PETSc GMRES solvers, there was no appreciable difference in the solution time for overall viscosity variations of $10^{9}$.

\subsection{Solver Scaling with Problem Size}

Multigrid, properly implemented, is particularly well suited for large scale problems because the solution time scales linearly with the number of unknowns. Figure 2 shows the scaling of the solution time for each of the methods, normalized by the number of unknowns and the solution time for the smallest problem. The times are the result of 


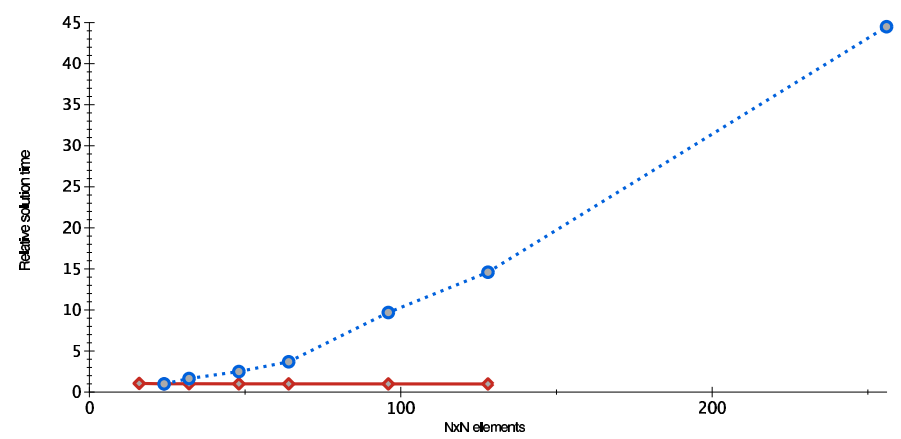

Fig. 2. Scaling of problem time with the number of velocity unknowns for multigrid (red) and GMRES (blue). The timings are normalized by the number of unknowns and scaled by the coarsest grid solution time. A flat curve is desirable since it indicates the work per unknown does not change with the size of the problem

averaging several runs with different viscosity laws. The multigrid scaling is very close to linear for the entire range of problem-sizes explored, whereas GMRES performance degrades quickly as the problem size increases.

\subsection{Parallel Efficiency}

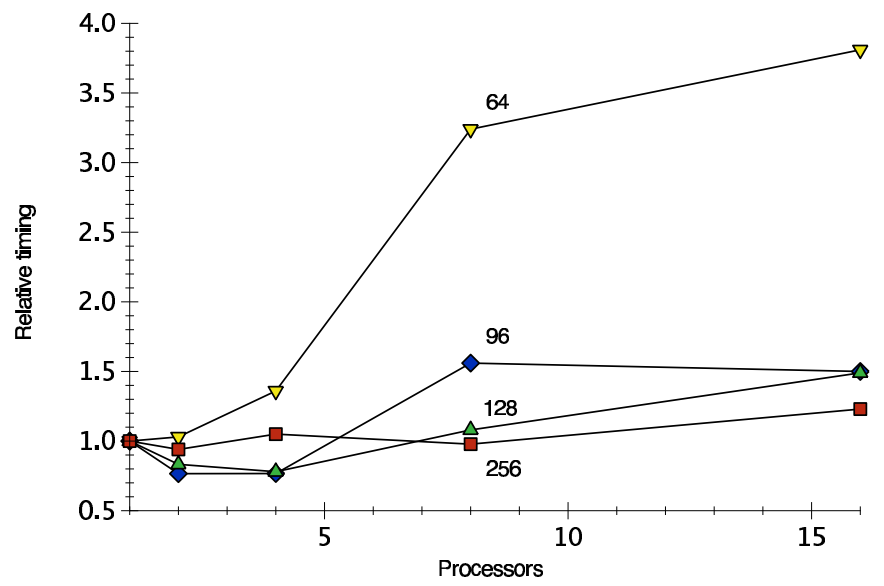

Fig. 3. Efficiency of the parallelization inherited from PETSc as a function of the number of processors. The timings are scaled to the solution time on a single processor in each case and then multiplied by the number of processors. Values below 1 indicate that the problem size is too large for efficient execution on a single processor, values significantly above 1 indicate inefficiencies in the parallel code - e.g. due to over-decomposition 
The parallel benchmarks were run on a distributed-memory cluster machine with low-latency communication. In figure 3 we plot the solution time scaled by the number of processors for parallel runs on different grid sizes. In this case it is clear that the efficiency of the parallelization using PETSc is extremely good, provided that the problem is sufficiently large to warrant decomposition. The timings are averaged over several runs, but the fact that the cluster was heavily loaded during these benchmarks introduced some scatter into the results which is evident in the figure.

\section{Discussion}

The abstraction of parallelism provided by building a code upon the PETSc libraries provides significant benefits in code readability, compactness, flexibility and maintainability. The parallel performance of the resulting code scales very well on a typical supercomputing cluster. The only issue we have found to date is that the PETSc solvers need to be chosen carefully to reproduce the efficiency of a solver written especially for the problem in hand. Further exploration of the issue of general PETSc multigrid solvers for this problem is needed.

\section{References}

Barazangi, M., and Dorman, J., (1969), World seismicity maps compiled from ESSA, Coast and Geodetic Survey, epicenter data 1961-1967, Bull. Seism. Soc. Am. 59, 369-380.

Karato, S.-I. and Wu, P., (1993), Rheology of the upper mantle: A synthesis, Science, 260, 771-778. McKenzie, D.P., (1977), Surface deformation, gravity anomalies, and convection, Geophys. J. R. Astr. Soc., 48, 211-238.

Moresi, L., Mühlhaus, H.-B., Dufour, F., Viscoelastic formulation for modelling of plate tectonics, In Bifurcation and localization in soils and rocks 99. Mühlhaus, Dyskin, A. and Pasternak, E. (ed), (Balkema, Rotterdam, 2001)

Moresi, L., Solomatov, V.S., (1995), Numerical investigations of 2D convection in a fluid with extremely large viscosity variations, Phys. Fluids, 7, 2154-2162

Moresi, L., Solomatov, V.S., (1998), Mantle convection with a brittle lithosphere: thoughts on the global tectonics styles of the Earth and Venus, Geophys. J. Int., 133, 669-682.

Moresi, L., Dufour, F., Mühlhaus, H.-B. (2002) Mantle convection models with viscoelastic/brittle lithosphere: Numerical methodology and plate tectonic modeling. Pure Appl. Geophys., 159, 2335-2356.

Moresi, L., Dufour, F., Mühlhaus, H.-B. (2003) A lagrangian integration point finite element method for large deformation modeling of viscoelastic geomaterials. J. Comput. Phys., 184, 476-497.

Moresi, L., Zhong, S.J. , Gurnis, M. (1996), The accuracy of finite element solutions of Stokes' flow with strongly varying viscosity. Phys. Earth Planet. Inter., 97, 83-94.

Solomatov, V.S., (1995), Scaling of temperature- and stress-dependent viscosity convection, Phys. Fluids, 7, 266-274.

Sulsky, D., Zhou, S.-J., Schreyer, H.L., (1995), Application of a particle-in-cell method to solid mechanics, Comput. Phys. Commun. 87, 236-252.

Watts, A.B., Bodine, J.H., Ribe, N.M., (1980), Observations of flexure and the geological evolution of the Pacific Basin. Nature, 283, 532-537. 